\title{
Imaging of nanoislands in coherent grazing-incidence small-angle x-ray scattering experiments
}

\author{
A. V. Zozulya, ${ }^{1}$ O. M. Yefanov, ${ }^{1}$ I. A. Vartanyants,${ }^{1, *}$ K. Mundboth,${ }^{2,3}$ C. Mocuta, ${ }^{2}$ T. H. Metzger,${ }^{2}$ J. Stangl, ${ }^{3}$ \\ G. Bauer, ${ }^{3}$ T. Boeck, ${ }^{4}$ and M. Schmidbauer ${ }^{4}$ \\ ${ }^{1}$ HASYLAB at DESY, Notkestrasse 85, D-22607 Hamburg, Germany \\ ${ }^{2}$ ESRF, 6 rue Jules Horowitz, F-38043 Grenoble, France \\ ${ }^{3}$ Institut für Halbleiterphysik, Johannes Kepler Universität Linz, A-4040 Linz, Austria \\ ${ }^{4}$ Leibniz Institute for Crystal Growth, Max-Born-Straße 2, D-12489 Berlin, Germany
}

(Received 11 June 2008; published 19 September 2008)

\begin{abstract}
Coherent $\mathrm{x}$-ray scattering in a grazing-incidence geometry was used to image SiGe nanoislands grown by liquid phase epitaxy on $\mathrm{Si}(001)$. Due to their narrow size distribution, identical shape, and orientation, the total scattered intensity obtained in this geometry represents a coherent diffraction pattern from an average island. Iterative phase retrieval techniques were used for the reconstruction of the projected electron density of hundred nanometer size islands. So far other x-ray imaging methods do not achieve the obtained spatial resolution of $10-15 \mathrm{~nm}$ for islands of this size.
\end{abstract}

DOI: 10.1103/PhysRevB.78.121304

PACS number(s): 61.05.cf, 68.65.-k, 68.37.Yz

Quantum dots (QD) can serve as building blocks for nanotechnological applications. ${ }^{1}$ Progress in nanoscience and nanotechnology requires tools to characterize the structure of such objects both on mesoscopic and atomic levels. These tools will become increasingly important for nanostructures embedded into electronic devices. X-ray diffraction methods are especially well suited for the determination of the shape, composition, and strain distribution in nanostructures. ${ }^{2-4}$ In a conventional approach using $\mathrm{x}$-ray scattering methods the structural information (shape, strain, and composition) is obtained indirectly by fitting the measured diffraction patterns with simulated ones derived from realistic modeling of the nanostructures. In this Rapid Communication we propose to use phase retrieval techniques ${ }^{5,6}$ for high-resolution imaging of nanostructures with a model independent approach. We determine the electron density of epitaxial SiGe nanoislands using coherent scattering in a grazing-incidence small-angle x-ray scattering (GISAXS) geometry. Here the crystal lattice, and hence strain fields, play no role and the scattered intensity distribution depends only on the electron-density distribution, shape, and size. The GISAXS geometry provides the additional advantage that due to the limited penetration into the substrate, the sensitivity to the QDs is enhanced considerably.

One of the strongest limitations in conventional imaging of nanostructures using $\mathrm{x}$ rays is the ultimate spatial resolution that can be achieved. Developments in x-ray optics have opened the possibility to perform measurements with $100 \mathrm{~nm}$ resolution and below. ${ }^{7-11}$ However, reaching nanometer resolution in the hard $\mathrm{x}$-ray range is still a significant challenge at presently available high-brilliance synchrotron sources. From that respect alternative approaches that provide the possibility to reach this resolution limit are very attractive from a practical point of view. Here we demonstrate that the application of coherent scattering in a GISAXS geometry yields a considerable enhancement of the scattered signal that finally provides an opportunity to reach tens of nanometers resolution for islands of order hundred nanometer in size.
The coherent $\mathrm{x}$-ray diffractive imaging (CXDI) microscopy technique was recently developed by several groups. ${ }^{12-16}$ The method is based on the following principle: if an object of finite size is coherently illuminated and its diffraction pattern is sufficiently sampled then it can be uniquely inverted ${ }^{17}$ to obtain an image of this object. As soon as no lenses are required in this imaging technique (therefore also known as lensless microscopy) it allows, at least in principle, to overcome the resolution limits of conventional microscopy techniques and to obtain diffraction-limited images of a sample using phase retrieval techniques. ${ }^{5,6}$ This is especially important for imaging samples smaller than $100 \mathrm{~nm}$.

Direct application of phase retrieval methods for reconstruction of the shape of the islands from coherent GISAXS data is, however, complicated by the fact that kinematical scattering theory (the first Born approximation) fails to describe the scattering process correctly. Instead, the distortedwave Born approximation (DWBA) is required. ${ }^{18}$ For nanoislands grown on the surface of a substrate, the total DWBA amplitude can be expressed as a coherent sum of four scattered amplitudes that takes into account multiplescattering effects. ${ }^{19,20}$ A detailed theoretical analysis of the contribution of each scattering channel at different scattering angles to the total scattering intensity was given in Ref. 21 (for experimental observations see Ref. 22). It was demonstrated that at an angle of incidence equal to the critical angle $\left(\alpha_{c}\right)$ of total external reflection from the substrate and for exit angles measured in the range above the critical angle the main contribution to the DWBA amplitude is determined by a coherent sum of two scattering channels: (i) the specular reflection from the surface followed by scattering from an island and (ii) purely kinematical scattering from an island (Fig. 1). It was demonstrated that at these specific scattering conditions, the GISAXS diffraction pattern can be inverted by conventional phase retrieval algorithms providing a reliable image of the two-dimensional (2D) projection of the electron density of a nanometer sized island on the plane perpendicular to the direction of the incoming beam.

The total DWBA amplitude with two scattering channels can be written in the form ${ }^{23} A_{\mathrm{DWBA}}\left(\mathbf{q}_{\|}, q_{z}\right)$ $\approx R\left(\alpha_{i}\right) A_{\text {kin }}\left(\mathbf{q}_{\|}, q_{z}\right)+A_{\text {kin }}\left(\mathbf{q}_{\|}, q_{z}+q_{0}\right)$, where $R\left(\alpha_{i}\right)$ is the am- 


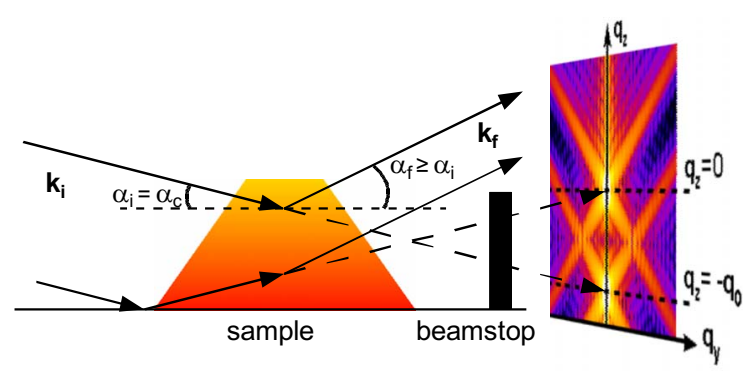

FIG. 1. (Color online) Schematic of the experiment in GISAXS geometry on a single island in the form of a truncated pyramid with a square base. Two dominant scattering channels (the specular reflection from the surface followed by scattering from an island, and purely kinematical scattering) exist in the conditions when $\alpha_{i}=\alpha_{c}$ and $\alpha_{f} \geq \alpha_{c}$. Note that the origin for two scattering amplitudes is shifted in reciprocal space by the value $q_{z}=-q_{0}$. The lower half of the reciprocal space $q_{z}<0$ is blocked by a beamstop and is not measured in our experiment.

plitude of the specularly reflected wave at the incidence angle $\alpha_{i}, A_{\text {kin }}\left(\mathbf{q}_{\|}, q_{z}\right)$ denotes the kinematically scattered amplitude. For small angles the $z$ component of the scattering vector is given by $q_{z}=k\left(\alpha_{f}-\alpha_{i}\right)$, where $k$ denotes the length of the wave vector, $\alpha_{f}$ is an exit angle and $q_{0}=2 k \alpha_{i}$. For the incidence angle, $\alpha_{i}=\alpha_{c}$ within a good approximation, $\left|R\left(\alpha_{i}\right)\right| \sim 1$ and $\arg \left[R\left(\alpha_{i}\right)\right] \sim 0$, that results in a total scattered intensity

$$
\begin{aligned}
I_{\text {DWBA }}\left(\mathbf{q}_{\|}, q_{z}\right)= & I_{\text {kin }}\left(\mathbf{q}_{\|}, q_{z}\right)+I_{\text {kin }}\left(\mathbf{q}_{\|}, q_{z}+q_{0}\right) \\
& +2 \operatorname{Re}\left[A_{\text {kin }}\left(\mathbf{q}_{\|}, q_{z}\right) A_{\text {kin }}^{*}\left(\mathbf{q}_{\|}, q_{z}+q_{0}\right)\right] .
\end{aligned}
$$

Here the first term is purely kinematical with its maximum intensity value at $q_{z}=0$ that corresponds to the exit angle $\alpha_{f}=\alpha_{i}$ and gives the major contribution to the scattered intensity in the upper half of the reciprocal space $q_{z}>0$. The contribution of the second channel [second term in Eq. (1)] in this part of the reciprocal space is significantly reduced (by two orders of magnitude) due to its shift in $q_{z}$ direction by the momentum-transfer value $q_{0}$ (Fig. 1) and can be safely neglected. Based on this analysis the inversion of diffraction data should give the correct shape and electron density of an island for these conditions. Small artifacts can be produced by an additional contribution of the third interference term in Eq. (1) that is one order of magnitude smaller than the leading first term.

We performed a series of measurements to validate the predictions of the theoretical analysis. ${ }^{21}$ First test experiments were performed at the BW4 small-angle scattering beamline at HASYLAB. ${ }^{24}$ Final experiments were performed at the ID01 beamline of the European Synchrotron Radiation Facility (ESRF) in Grenoble. The x-ray energy was set to $8 \mathrm{keV}$. The incidence angle was taken equal to the critical angle for the $\mathrm{Si}$ substrate which corresponds to $\alpha_{i}$ $=\alpha_{c}=0.224^{\circ}$ for our incident energy. GISAXS data were recorded using a Princeton Instruments charge-coupled device (CCD) area detector, with $55.5 \mu \mathrm{m}$ pixel size. The CCD detector was placed in vacuum inside a flight tube to avoid air scattering, and hence obtain a clean scattering pattern.
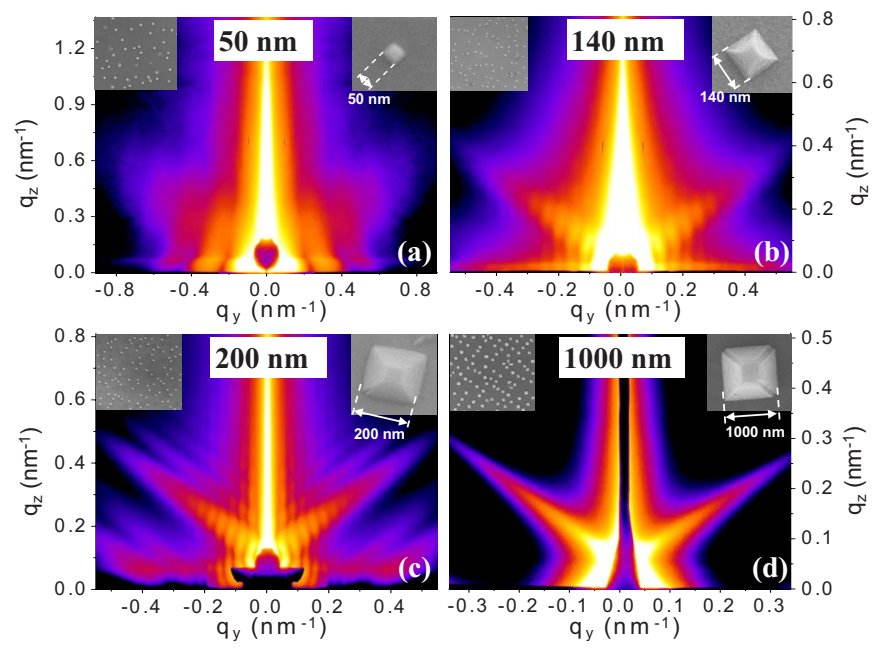

FIG. 2. (Color online) GISAXS diffraction patterns measured for different island sizes (a) $50 \mathrm{~nm}$, (b) $140 \mathrm{~nm}$, (c) $200 \mathrm{~nm}$, and (d) $1000 \mathrm{~nm}$. SEM images of the samples with different magnification are presented in the insets. In panels (a)-(c) two diffraction patterns measured with a large beamstop and a small beamstop are superimposed. Each diffraction pattern is background subtracted. The logarithmic color scale corresponds to a dynamic range of $10^{5}-10^{6}$.

We used SiGe islands of different average size grown by liquid phase epitaxy ${ }^{3}$ (LPE) as our model samples. All islands were grown on a (001) Si surface and exhibit a truncated pyramidal shape with a square base (see insets in Fig. 2). In addition they typically have a narrow size distribution $[\sim 10 \%$ full width at half maximum (FWHM) $]$ and the same crystallographic orientation on a $\mathrm{Si} \operatorname{surface}^{3}$ (Fig. 2). The direction of the incident beam was chosen to be parallel to the pyramid base edges which are oriented along the $\langle 110\rangle$ directions. In GISAXS geometry with an incoming beam of a few hundreds of microns size a large number of typically $10^{6} \mathrm{SiGe}$ islands are illuminated simultaneously.

In the case of a fully coherent beam the total scattered amplitude from $N$ islands is given by $A(\mathbf{q})$ $=\sum_{i=1}^{N} A_{i}(\mathbf{q}) \exp \left(i \mathbf{q} \mathbf{R}_{i}\right)$, where $A_{i}(\mathbf{q})$ is the coherent scattering amplitude from an individual island, ${ }^{25} \mathbf{R}_{i}$ is the position of that island on the surface of the sample, and $\mathbf{q}$ is the scattering vector. For a narrow size distribution of islands the scattered intensity is given by the following expression

$$
I(\mathbf{q})=N I_{a v}^{\mathrm{coh}}(\mathbf{q})\left[1+\frac{1}{N} \sum_{i \neq j} \exp \left[i \mathbf{q}\left(\mathbf{R}_{i}-\mathbf{R}_{j}\right)\right]\right],
$$

where $I_{a v}^{\mathrm{coh}}(\mathbf{q})$ is the coherently scattered intensity from the average electron density of an island. From Eq. (2) it follows that for identical islands a small diffracted intensity from an individual island $I_{a v}^{\mathrm{coh}}(\mathbf{q})$ is significantly enhanced by the contribution from $N$ illuminated islands. In the case of a partially coherent beam the second interference term in Eq. (2) gives rise to a speckle pattern with the speckle size determined by the coherence length of the incoming beam. For the conditions of our experiment the incoming beam has a lateral coherence length of about $5 \mu \mathrm{m}$ in both horizontal and vertical directions. In this case each island of a few nanometers size is coherently illuminated. Due to the large coherence length, 
speckles originating from a random distribution of islands were not observed because the speckle size was actually below the pixel size of the detector used. Finally, for the conditions of our experiment the scattered intensity represents an enhancement by about a factor of $10^{6}$ of the coherently scattered signal from an average island in the ensemble. Using an unfocused beam at ID01 at ESRF the incoming photon flux per average island multiplied by the number of islands can be estimated as $10^{10}$ photons/s which is $10^{13}$ photons integrated over the total data-acquisition time.

Diffraction patterns measured for four different samples with island base sizes of 50, 140, 200, and $1000 \mathrm{~nm}$ are shown in Fig. 2. The sample to detector distance was $1.5 \mathrm{~m}$ for the $50 \mathrm{~nm}$ islands, $2.5 \mathrm{~m}$ for the $140 \mathrm{~nm}$ and the $200 \mathrm{~nm}$ islands, and $4 \mathrm{~m}$ for the $1000 \mathrm{~nm}$ islands, resulting in a resolution per detector pixel of $\Delta q=1.5 \times 10^{-3}, 0.9 \times 10^{-3}$, and $0.56 \times 10^{-3} \mathrm{~nm}^{-1}$, respectively. As the dynamic range of these diffraction patterns is much larger than that of the CCD detector, the measurements were performed in a few steps by using a series of "beamstops" blocking the very intense part of the signal. The diffraction patterns measured with these different beamstops were superimposed to produce diffraction patterns with their full dynamic range as shown in Figs. 2(a)-2(c). Strong crystal truncation rods (CTR) emerging from $\{111\}$ side facets of the SiGe nanoislands can be clearly seen. The maximum of the intensity distribution is pointing in the directions normal to the $\{111\}$ island facets. In Fig. 2 one recognizes that the intensity along the CTRs is oscillating due to the coherent illumination. The period of these oscillations is determined by the distance from the $\{111\}$ facets of the $\mathrm{SiGe}$ islands to the opposite edges. ${ }^{23}$ This period decreases for larger islands and finally disappears for the islands of $1000 \mathrm{~nm}$ size. Careful examination of the sample [see scanning electron microscopy (SEM) image in the inset in Fig. 2(d)] shows that interference fringes from individual islands are most probably destroyed due to a difference in the shape of individual islands rather than by a reduced coherence of the incoming beam. Taking into account that the spatial resolution is determined by the maximum value of the scattering vector measured in the experiment, we estimate the obtained resolution by analyzing the drop of interference fringes along the $\langle 111\rangle$ flares of each diffraction pattern. This analysis yields a resolution value of $15 \mathrm{~nm}$ for the islands of 140 and $200 \mathrm{~nm}$ size, and $10 \mathrm{~nm}$ for $50 \mathrm{~nm}$ islands. ${ }^{26}$

To invert the coherent GISAXS patterns shown in Fig. 2 we used an iterative phase retrieval approach. We emphasize that for this method of phase retrieval no starting model of the sample is used for fitting the data. In the first step the missing phases are taken as a random set and are combined with the amplitudes in reciprocal space. Then a Fourier transform is applied back and forth with specific constraints used in both real and reciprocal spaces. In reciprocal space, as a natural constraint, the measured diffraction pattern is used and in the real space a finite support and the positivity of the electron density are used. To test the reproducibility, the calculations are repeated with another set of starting random phases. To avoid stagnation we used an alternation of two algorithms: error reduction (ER: 50 cycles) and hybrid input output $^{5}$ (HIO: 100 cycles) having about 6000 total cycles for each set of random phases. The actual parameters of these
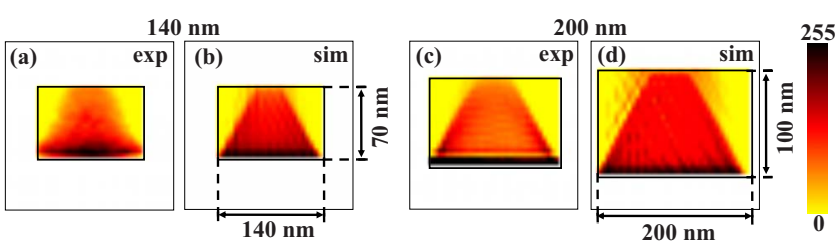

FIG. 3. (Color online) [(a), (c)] Results of the reconstruction of the island electron-density projections for 140 and $200 \mathrm{~nm}$ islands obtained from experimental GISAXS data as shown in Fig. 2. [(b), (d)] For comparison, the reconstruction of a single island electron density obtained from a simulated GISAXS pattern is also shown. The size of the rectangular support region used in each reconstruction is indicated by a black line. The color gradient in arbitrary units is shown in the inset. The length scale is the same for all four figures but is different in vertical and horizontal directions.

algorithms and constraints used in the reconstruction of our coherent GISAXS data are similar to the ones used in Refs. 14 and 21.

Results of the reconstruction of the electron density projection for islands of 140 and $200 \mathrm{~nm}$ size are presented in Fig. 3. The electron density of the reconstructed islands shown in this figure is a result of averaging of a few of the best reconstructions with different sets of starting random phases. In this way we increase the fidelity of the obtained result. For comparison, a reconstruction of the island shape obtained from a simulated GISAXS pattern (calculated with the IsGISAXS program ${ }^{27}$ ) is also shown in Figs. 3(b) and 3(d). As a model for GISAXS calculations, a single island in the form of a truncated pyramid with a square base was considered. The height $h$ of the island in the calculations was chosen by taking into account the aspect ratio of $h / w=0.5$ obtained from an SEM, where $w$ corresponds to the base size of 140 or $200 \mathrm{~nm}$, respectively. From these figures we see that for our scattering conditions the projection of the electron density, the shape, and the size of the islands are well reproduced. The average base size of the $200 \mathrm{~nm}$ islands in reconstruction turned out to be smaller $(\sim 185 \mathrm{~nm})$. It can also be observed that for the measured islands of $140 \mathrm{~nm}$ size the electron density of the side $\{111\}$ facets are more smeared out in comparison to simulated ones. This is well correlated with the SEM image in the inset in Fig. 2(b) showing increased roughness on the facets of these islands. Unfortunately the rather low signal-to-noise ratio present in our measured diffraction patterns of $50 \mathrm{~nm}$ islands [Fig. 2(a)] made the reconstruction of these islands difficult.

In conclusion, we have demonstrated how coherent GISAXS technique can be used to image nanometer sized objects. The high resolution of about $10-15 \mathrm{~nm}$ which is obtained in our experiment is strongly related to the enhancement of the scattered signal due to the uniformity of our islands. It is important to note that this approach does not depend on the crystalline structure of such an object and can, in principle, be applied to any material system. It may be applied, for example, to embedded structures with a different refractive index from the host material more readily than electron-scattering techniques that rely strongly on sample preparation. An important extension of this technique is its application to the collection of three-dimensional (3D) data 
measured by rotating the sample around its axis perpendicular to the substrate surface, while maintaining grazingincidence beam conditions. ${ }^{23}$ Such data can be used for the reconstruction of the $3 \mathrm{D}$ electron density of nanoislands. An important future application of this technique could be in its combination with strongly focused beams, ${ }^{7-11}$ or in experiments performed with $\mathrm{x}$-ray free electron lasers ${ }^{28,29}$ (XFEL). In this case the strong gain of the incoming photon flux (about five orders of magnitude for the focusing devices, and $10^{12}$ photons per single femtosecond pulse for XFEL) on a single island below $100 \mathrm{~nm}$ in size can be expected to pro- vide an effective tool for investigating nanostructures with nanometer spatial resolution.

\section{ACKNOWLEDGMENTS}

The authors thank I. Snigireva for support in recording and analyzing the SEM images. Part of this work was supported by the FWF Vienna (SFB025). A.V.Z., O.M.Y and I.A.V. acknowledge the interest and support of J. Schneider and E. Weckert during the work on this project and A. Mancuso for a careful reading of the manuscript.
*Corresponding author; ivan.vartaniants@desy.de

${ }^{1}$ V. A. Shchukin, N. N. Ledentsov, and D. Bimberg, Epitaxy of Nanostructures (Springer, New York, 2004).

${ }^{2}$ J. Stangl, V. Holy, and G. Bauer, Rev. Mod. Phys. 76, 725 (2004).

${ }^{3}$ M. Schmidbauer, X-Ray Diffuse Scattering from Self-Organized Mesoscopic Semiconductor Structures, Springer Tracts in Modern Physics Vol. 199 (Springer, Berlin, 2004).

${ }^{4}$ U. Pietsch, V. Holy, and T. Baumbach, High-Resolution X-Ray Scattering from Thin Films to Lateral Nanostructures, Advanced Texts in Physics, 2nd ed. (Springer, Berlin, 2004).

${ }^{5}$ J. R. Fienup, Appl. Opt. 21, 2758 (1982).

${ }^{6}$ V. Elser, J. Opt. Soc. Am. A 20, 40 (2003).

${ }^{7}$ C. G. Schroer, O. Kurapova, J. Patommel, P. Boye, J. Feldkamp, B. Lengeler, M. Burghammer, C. Riekel, L. Vincze, A. van der Hart, and M. Küchler, Appl. Phys. Lett. 87, 124103 (2005).

${ }^{8}$ O. Hignette, P. Cloetens, G. Rostaing, P. Bernard, and C. Morawe, Rev. Sci. Instrum. 76, 063709 (2005).

${ }^{9}$ H. C. Kang, J. Maser, G. B. Stephenson, C. Liu, R. Conley, A. T. Macrander, and S. Vogt, Phys. Rev. Lett. 96, 127401 (2006).

${ }^{10}$ B. Nöhammer, C. David, M. Burghammer, and C. Riekel, Appl. Phys. Lett. 86, 163104 (2005).

${ }^{11}$ M. Hanke, M. Dubslaff, M. Schmidbauer, T. Boeck, S. Schoeder, M. Burghammer, C. Riekel, J. Patommel, and C. Schroer, Appl. Phys. Lett. 92, 193109 (2008).

${ }^{12}$ J. Miao, P. Charalambous, J. Kirz, and D. Sayre, Nature (London) 400, 342 (1999).

${ }^{13}$ M. Pfeifer, G. Williams, I. Vartanyants, R. Harder, and I. Robinson, Nature (London) 442, 63 (2006).

${ }^{14}$ I. A. Vartanyants, I. K. Robinson, J. D. Onken, M. A. Pfeifer, G. J. Williams, F. Pfeiffer, H. Metzger, Z. Zhong, and G. Bauer, Phys. Rev. B 71, 245302 (2005).

${ }^{15}$ J. Miao, C.-C. Chen, C. Song, Y. Nishino, Y. Kohmura, T. Ishikawa, D. Ramunno-Johnson, T.-K. Lee, and S. H. Risbud, Phys. Rev. Lett. 97, 215503 (2006).

${ }^{16}$ H. N. Chapman, A. Barty, S. Marchesini, A. Noy, C. Cui, M. R. Howells, R. Rosen, H. He, J. C. H. Spence, U. Weierstall, T. Beetz, C. Jacobsen, and D. Shapiro, J. Opt. Soc. Am. A 23, 1179 (2006).
${ }^{17}$ R. H. T. Bates, Optik (Stuttgart) 61, 247 (1982).

${ }^{18}$ S. K. Sinha, E. B. Sirota, S. Garoff, and H. B. Stanley, Phys. Rev. B 38, 2297 (1988).

${ }^{19}$ M. Rauscher, R. Paniago, H. Metzger, Z. Kovats, J. Domke, J. Peisl, H.-D. Pfannes, J. Schulze, and I. Eisele, J. Appl. Phys. 86, 6763 (1999).

${ }^{20}$ R. Lazzari, J. Appl. Crystallogr. 35, 406 (2002).

${ }^{21}$ I. A. Vartanyants, D. Grigoriev, and A. Zozulya, Thin Solid Films 515, 5546 (2007).

${ }^{22}$ M. Schmidbauer, D. Grigoriev, M. Hanke, P. Schäfer, T. Wiebach, and R. Köhler, Phys. Rev. B 71, 115324 (2005).

${ }^{23}$ I. A. Vartanyants, A. V. Zozulya, K. Mundboth, O. M. Yefanov, M. I. Richard, E. Wintersberger, J. Stangl, A. Diaz, C. Mocuta, T. H. Metzger, G. Bauer, T. Boeck, and M. Schmidbauer, Phys. Rev. B 77, 115317 (2008).

${ }^{24}$ I. A. Vartanyants, A. V. Zozulya, A. Veligzhanin, and S. V. Roth, in HASYLAB Annual Report 2006, edited by W. Caliebe, W. Drube, and J. R. Schneider (HASYLAB at DESY, Hamburg, 2006), Pt. 1, p. 253.

${ }^{25}$ In the case of GISAXS scattering each amplitude $A_{i}(\boldsymbol{q})$ corresponds to a total DWBA amplitude.

${ }^{26}$ We want to note here that the FWHM of the size and orientation distribution of the islands should be much less than $50 \%$. The broader size and orientation distribution will smear interference fringes (see Fig. 2(d)) and will reduce achievable resolution values.

${ }^{27}$ ISGISAXS Program, http://www.insp.upmc.fr/axe2/Oxydes/ IsGISAXS/isgisaxs.htm

${ }^{28}$ H. N. Chapman, A. Barty, M. J. Bogan, S. Boutet, M. Frank, S. P. Hau-Riege, S. Marchesini, B. W. Woods, S. Bajt, W. H. Benner, R. A. London, E. Plönjes, M. Kuhlmann, R. Treusch, S. Düsterer, Th. Tschentscher, J. R. Schneider, E. Spiller, Th. Möller, C. Bostedt, M. Hoener, D. A. Shapiro, K. O. Hodgson, D. van der Spoel, F. Burmeister, M. Bergh, C. Caleman, G. Huldt, M. M. Seibert, F. R. N. C. Maia, R. W. Lee, A. Szöke, N. Timneanu, and J. Hajdu, Nat. Phys. 2, 839 (2006).

${ }^{29}$ I. A. Vartanyants, I. K. Robinson, I. McNulty, C. David, P. Wochner, and Th. Tschentscher, J. Synchrotron Radiat. 14, 453 (2007). 